\title{
Pediatric Inflammatory Multisystem Syndrome Temporally Associated with SARS-CoV-2: a New Challenge amid the Pandemic
}

\author{
Sherly Lawrensia ${ }^{1}$ (D) - Joshua Henrina ${ }^{1,2}$ (D) Ellen Wijaya ${ }^{3}$. Leonardo Paskah Suciadi ${ }^{2}$. \\ Aninka Saboe ${ }^{4}$. Charlotte Johanna Cool ${ }^{4}$
}

Accepted: 15 October 2020 / Published online: 22 October 2020

(C) Springer Nature Switzerland AG 2020

\begin{abstract}
Initially, SARS-CoV-2 infection had been reported as a relatively mild case in children than in adults. Nevertheless, recent evidence found that a subset of children then developed a significant systemic inflammatory response that resembles atypical/ typical Kawasaki's disease (KD) and toxic shock syndrome. This novel clinical syndrome later identified as pediatric inflammatory multisystem syndrome temporally associated with SARS-CoV-2 (PIMS-TS). In contrast with KD, PIMS-TS appears to occur in children at an older age with a predominance of gastrointestinal symptoms, hemodynamic instability, and myocardial dysfunction. However, the exact pathomechanism remains to be understood. Nevertheless, the post-viral immunological reaction is postulated to be the underlying mechanistic underpinnings. The multifaceted nature of the PIMS-TS' course underlines the need for early recognition and multispecialty care and management.
\end{abstract}

Keywords PIMS-TS · SARS-CoV-2 $\cdot$ COVID-19 $\cdot$ MIS-C $\cdot$ Hyperinflammatory syndrome

\section{Introduction}

The coronavirus disease 2019 (COVID-19) pandemic caused by the severe acute respiratory syndrome coronavirus 2 (SARS-CoV-2) infection has rapidly spread throughout the world. As of 14 September 2020, data from the World Health Organization (WHO) showed that COVID-19 has affected almost twenty-nine million people and is attributable to more than 917.000 deaths [1]. Previously, SARS-CoV-2

This article is part of the Topical Collection on Covid-19

Sherly Lawrensia

sherlylawrensia@hotmail.com

Joshua Henrina

joshuahenrina@gmail.com

Ellen Wijaya

Wijaya.ellen@gmail.com

Leonardo Paskah Suciadi

Be.bakestreet@gmail.com

Aninka Saboe

Aninka.saboe@unpad.ac.id

Charlotte Johanna Cool

Charlotte.dewantara@gmail.com infection has been reported as a relatively mild case in children than in adults [2]. Nevertheless, recent evidence revealed that a subset of children might develop significant complications of a systemic inflammatory response that resembles atypical/ typical Kawasaki's disease (KD) and toxic shock syndrome [3]. This novel clinical syndrome has been later identified as pediatric inflammatory multisystem syndrome temporally associated with SARS-CoV-2 (PIMS-TS). Following the emergence of PIMS-TS, the WHO, Centers for Disease Control

1 School of Medicine and Health Sciences, Atma Jaya Catholic University of Indonesia, Jl. Pluit Raya No. 2, RT 21/RW 08, Penjaringan, Kota Jakarta Utara, Daerah Khusus Ibukota Jakarta 14440 , Indonesia

2 Siloam Heart Institute, Siloam Hospital Kebon Jeruk, Jl. Perjuangan No.8, RT.14/RW.10, Kb. Jeruk, Kec. Kb. Jeruk, Kota Jakarta Barat, Daerah Khusus Ibukota Jakarta 11530, Indonesia

3 Department of Pediatrics, School of Medicine and Health Sciences, Atma Jaya Catholic University of Indonesia, Jl. Pluit Raya No. 2, RT 21/RW 08, Penjaringan, Kota Jakarta Utara, Daerah Khusus Ibu kota Jakarta 14440, Indonesia

4 Cardiology and Vascular Department, Padjadjaran University, Jl. Professor Eyckman No. 38, Pasteur, Kec. Sukajadi, Kota Bandung, Jawa Barat 40161, Indonesia 
and Prevention (CDC), and the European Centre for Disease Prevention and Control (ECDPC) had provided the case definitions [4-6]. Ever since the issuance of various case definitions from these organizations above, several case series and case reports from various regions were published to explain and discuss the clinical manifestations up to outcomes of patients with PIMS-TS. Therefore, this review will be focused on describing the epidemiology and clinical manifestation in PIMS-TS.

\section{Epidemiology}

In April 2020, the National Health Service of the UK raised the alarm of children with serious hyperinflammatory syndrome, which some of them had positive lab-confirmed COVID-19. Since then, other countries started to report the same clinical syndrome (France, USA, Italy, Spain, and India) [7-11]. Compared to KD, there are distinctive epidemiological characteristics of PIMS-TS. PIMS-TS appeared to occur among children of all racial and ethnic backgrounds in contrast with KD, which mostly affects East-Asian ethnicity. However, the observation of case reports and series found a higher proportion of African and Asian ancestry in PIMS-TS. Based on the age group, PIMS-TS predominantly affects children with older age groups in comparison with typical $\mathrm{KD}$ (average age group around 9-15 years versus $2-5$ years, respectively) [3, 12]. Table 1 depicts the proportion of demographic characteristics, including age, gender, and ethnicity among children with PIMS-TS from case reports/series reported [2, 3, 7-11, 13-20].

\section{Case Definition}

Following the alert from NHS England and the UK Pediatric Intensive Care Society regarding unexplained multisystem inflammatory syndrome cases in the UK, on the 1 st of May 2020, the Royal College of Paediatrics and Child Health $(\mathrm{RCPCH})$ published a guidance and case definition related to this illness [6]. Since then, the WHO and CDC also developed definitions for this relatively new illness. Diagnostic criteria for this syndrome are presented in Table 2 [4-6].

\section{Proposed Mechanism of PIMS-TS}

Although PIMS-TS may indeed be associated with SARSCoV-2 infection, the pathophysiology of PIMS-TS remains elusive. Nevertheless, some evidence and reports suggest several mechanisms of this new clinical entity. The first possible hypothesis is that PIMS-TS is an immunologically mediated pathogenesis or a post-infectious process caused by nonneutralizing $\operatorname{IgG}$ antibody through antibody-dependent enhancement (ADE) [7, 19, 21]. The reported cases of PIMSTS emerged after the peak of SARS-CoV-2 infection in some countries. They found that most patients were more often had a positive test for antibody to SARS-CoV-2 than for the virus using nasopharyngeal RT-PCR, which raises the possibility of the involvement of acquired immunity aberrant development $[3,21]$. This evidence is supported by the finding from a large multicentre observational study among 78 children who fulfilled the case definition of PIMS-TS and were admitted to a pediatric intensive care unit (PICU) in the UK [12]. This study found that $96 \%$ of patients had SARS-CoV-2 antibodies but negative for its PCR. This result supports the evidence that PIMS-TS might not be an acute COVID-19 infection, but it is more likely a post-immunological reaction [12]. However, this ADE theory is still unclear because there has not been any report regarding the worsening of COVID-19 patients who received convalescent plasma [21].

The second proposed hypothesis of PIMS-TS is the cytokine storm, which is caused by the ability of coronaviruses to block types I and III interferon responses in patients with previously high viral load or patients who cannot handle the viral replication, thus resulting in delayed excessive cytokine storm [21].

Based on demographic characteristics, differences in racial distribution of those affected by PIMS-TS suggest another theory of whether there is a social effect or genetic susceptibility of a particular ethnicity and warrant future investigation. A hypothesis by Rowley might explain this ethnicity predisposition. African and Asian children in Europe and North America are thought to have earlier maturation of lung entry ports. Commensurately, the numbers of angiotensin-converting enzyme 2 receptors, which are essential for immunomodulation and necessary receptors for SARS-CoV-2 spike protein attachment, are higher in these two ethnicities compared to the Caucasian. Consequently, more lung cells are infected, and when these cells are destroyed, the spike protein is not eliminated, but release in large amounts. Subsequently, these highly immunogenic materials excite the immune system, culminating with an inflammatory storm. Another hypothesis is that the ethnicities above, while meeting the necessary sun exposure in their country of origin, may not be the case in Europe and North America.

Furthermore, an ethnically determined diet, which is a diet low in vitamin $\mathrm{D}$, is attributed to relative vitamin $\mathrm{D}$ deficiency. Meanwhile, vitamin D is known to play an active role in immune modulation. Thus, these factors made them more susceptible to hyperinflammation. Based on the age distribution of those affected by PIMS-TS, another hypothesis by Rowley explained that children $<2$ years of age have low levels of cellular receptor entry ports, which consequently only mildly affected by this disease or none at all [21]. 
Table 1 Demographic of PIMS-TS patients

\begin{tabular}{|c|c|c|c|c|c|}
\hline Country & Author & Gender $n / N(\%)$ & Age, median (IQR) & Ethnicity & $n / N(\%)$ \\
\hline \multirow[t]{13}{*}{ UK } & \multirow[t]{4}{*}{ Whittaker et al. [3] } & \multirow[t]{4}{*}{ Male 38/58 (66) } & \multirow[t]{4}{*}{$9(5.7-14)$} & African & $22 / 58(38)$ \\
\hline & & & & Asian & $18 / 58(31)$ \\
\hline & & & & White & $12 / 58(21)$ \\
\hline & & & & Middle Eastern & $6 / 58(10)$ \\
\hline & \multirow[t]{3}{*}{ Riphagen et al. [10] } & \multirow[t]{3}{*}{ Male 5/8 (62.5) } & \multirow[t]{3}{*}{$8(6-12.25)$} & Afro-Caribbean & $6 / 8(75)$ \\
\hline & & & & Asian & $1 / 8(12.5)$ \\
\hline & & & & Middle Eastern & $1 / 8(12.5)$ \\
\hline & \multirow[t]{4}{*}{ Ramcharan et al. [13] } & \multirow[t]{4}{*}{ Male 11/15 (73) } & \multirow[t]{4}{*}{$8.8(6.4-11.2)$} & Afro-Caribbean & $6 / 15(40)$ \\
\hline & & & & South Asian & $6 / 15(40)$ \\
\hline & & & & Mixed & 2/15 (13) \\
\hline & & & & Other & $1 / 15(7)$ \\
\hline & \multirow[t]{2}{*}{$\mathrm{Ng}$ et al. [14] } & \multirow[t]{2}{*}{ Male 2/3 (67) } & \multirow[t]{2}{*}{$(13-17)$} & \multirow{2}{*}{$\begin{array}{l}\text { Afro-Caribbean } \\
\text { Asian Indian }\end{array}$} & $2 / 3(67)$ \\
\hline & & & & & $1 / 3(33)$ \\
\hline \multirow[t]{8}{*}{ France } & Toubiana et al. [2] & Male 9/21 (43) & $7.9(3.7-16.6)$ & Afro-Caribbean & $24 / 42(57)$ \\
\hline & & & & Asian & 4/42 (10) \\
\hline & & & & Europe & $12(28)$ \\
\hline & & & & Middle Eastern & $2 / 42(5)$ \\
\hline & Belhadjer et al. [15] & Male 18/35 (52) & $10(2-6)$ & & \\
\hline & Blondiaux et al. [16] & Male 1/4 (25) & $9.5(7.5-11.25)$ & & \\
\hline & Pouletty et al. [17] & Male 8/16 (50) & $10(4.7-12.5)$ & & \\
\hline & Grimaud et al. [18] & Male 10/20 (50) & $10(2.9-15)$ & & \\
\hline Italy & Verdoni et al. [7] & Male 7/10 (70) & $7(5-7.75)$ & & \\
\hline Spain & Cabrero-Hernandez et al. [8] & Male 4/5 (80) & $10(9-13)$ & & \\
\hline USA & Kaushik et al. [11] & Male 20/33 (61) & $10(6-13)$ & Hispanic & $15 / 33(46)$ \\
\hline & & & & Black & $13 / 33(39)$ \\
\hline & & & & White & $3 / 33(9)$ \\
\hline & & & & Asian & $1 / 33(3)$ \\
\hline & & & & Other & $1 / 33(3)$ \\
\hline & Capone et al. [19] & Male 20/33 (61) & $8.6(5.5-12.6)$ & Black & $8 / 33(24)$ \\
\hline & & & & Asian & $3 / 33(9)$ \\
\hline & & & & White & $3 / 33(9)$ \\
\hline & & & & Other & $15 / 33(46)$ \\
\hline & & & & Unknown & $4 / 33(12)$ \\
\hline & Cheung et al. [20] & Male 8/17 (47) & $8(1.6-18)$ & Ashkenazi & $6 / 17(34)$ \\
\hline & & & & Jewish & $2 / 17(12)$ \\
\hline & & & & Non-Hispanic & $4 / 17(24)$ \\
\hline & & & & Hispanic & 4/17 (24) \\
\hline & & & & Black Asian & $1 / 17(6)$ \\
\hline India & Abdul Rauf [9] & Male 1/1 (100) & 5 & N/A & \\
\hline
\end{tabular}

\section{Fever}

Persistent fever is one of the criteria for PIMS-TS' case definition. The duration of fever for PIMS-TS is more than $24 \mathrm{~h}$ [5] or 3 days [4], in contrast with KD, which is $\geq 5$ days [22]. Fever is one of the clinical signs of inflammation, together with other inflammatory signs such as rash or conjunctivitis.
Almost all patients with PIMS-TS had a history of fever that occurred $4-8$ days $[7,11]$ before admission $[3,7,11,12,17$, 18]. The fever is mostly unrelenting with temperature ranging from 38 to $40^{\circ} \mathrm{C}$; meanwhile, seven cases of PIMS-TS reported by Pouletty et al. showed the temperature peaked over $40{ }^{\circ} \mathrm{C}[2,17]$. However, it is challenging to distinguish PIMS-TS and KD only based on fever characteristic since 
Table 2 Case definitions for pediatric inflammatory multisystem syndrome from the Royal College of Paediatrics and Child Health, World Health Organization, and Centers for Disease Control and Prevention

Royal College of Paediatrics and Child Health World Health Organization [4]

Centers for Disease Control and Prevention [5] (UK) [6]

- A child presenting with persistent fever, inflammation (neutrophilia, elevated CRP, and lymphopenia), and evidence of single- or multiorgan dysfunction (shock, cardiac, respiratory, renal, gastrointestinal, or neurological disorder) with additional features.

This may include children fulfilling full or partial criteria for Kawasaki disease*.
- Exclusion of any other microbial cause, including bacterial sepsis, staphylococcal or streptococcal shock syndromes, infections associated with myocarditis such as enterovirus (waiting for results of these investigations should not delay seeking expert advice).

- SARS-CoV-2 PCR testing may be positive or negative
- Children and adolescents $0-19$ years of age with fever $>3$ days AND two of the following:

a) Rash or bilateral non-purulent conjunctivitis or mucocutaneous inflammation signs (oral, hands, or feet).

b) Hypotension or shock

c) Features of myocardial dysfunction, pericarditis, valvulitis, or coronary abnormalities (including ECHO findings or elevated troponin/NT-proBNP)

d) Evidence of coagulopathy (by PT, PTT, elevated D-Dimers)

e) Acute gastrointestinal problems (diarrhea, vomiting, or abdominal pain)

- AND elevated markers of inflammation such as - AND no alternative plausible diagnoses ESR, C-reactive protein, or procalcitonin. kidney, respiratory, hematologic,
- An individual aged $<21$ years presenting with fever**, laboratory evidence of inflammation***, and evidence of clinically severe illness requiring hospitalization, with multisystem (>2) organ involvement (cardiac, gastrointestinal, dermatologic, or neurological)

- AND no other obvious microbial cause of inflammation, including bacterial sepsis, staphylococcal or streptococcal shock syndrome.

- AND evidence of COVID-19 (RT-PCR, antigen Additional comments: test, or serology positive), or likely contact with Some individuals may fulfill full or partial criteria patients with COVID-19

\begin{abstract}
- AND positive for current or recent SARS-CoV-2 infection by RT-PCR, serology, or antigen test; or COVID-19 exposure within the 4 week prior to the onset of symptoms for Kawasaki disease but should be reported if they meet the case definition for MIS-C

Consider MIS-C in any pediatric death with evidence of SARS-CoV-2 infection
\end{abstract}

*Criteria for Kawasaki disease include persistent fever and 4 of 5 principal clinical features: erythema and cracking of lips, strawberry tongue, and/or erythema of oral and pharyngeal mucosa; bilateral bulbar conjunctival injection without exudate; rash (maculopapular, diffuse erythroderma); erythema and edema of the hands and feet and/or periungual desquamation; and cervical lymphadenopathy; **Fever $>38.0 \mathrm{C}$ for $>24 \mathrm{~h}$ or report of subjective fever lasting $>24 \mathrm{~h}$; *** Laboratory evidence including, but not limited to $>1$ of the following: and elevated CRP level, ESR, fibrinogen, procalcitonin, D- dimer, ferritin, lactic acid dehydrogenase, or IL-6; elevated neutrophils; reduced lymphocytes; and low albumin

both have overlapping clinical features. The onset of fever is remarkably important since it helps recognize the course of disease and the follow-up after administering the treatment.

In $\mathrm{KD}$, administering intravenous immunoglobulin (IVIG) within 10 days of fever onset is effective in reducing the risk of coronary artery aneurysm [22]. Most fever in PIMS-TS abated after the initiation of IVIG (with a dose of $2 \mathrm{~g} / \mathrm{kg}$ ) $[17,23]$. However, in patients with IVIG-resistant fever, defined as persistence of fever at least $36 \mathrm{~h}$ and less than 7 days, the second infusion has to be given $[2,17]$.

Moreover, unexplained high fever, inflammation signs, and laboratory results in children with a history of COVID19 exposure, particularly in area with a high rate of COVID19 incidence or in the context of SARS-CoV-2 pandemic, should raise the suspicion for PIMS-TS. If the laboratory test (i.e., complete blood and differential count, CRP, ferritin, albumin) showed the presence of hyperinflammation, additional work-ups should be ordered to identify myocarditis (including troponin, NT-proBNP, and echocardiography) or cytokine storm syndrome (lactate dehydrogenase, fibrinogen, D-dimer, PTT, INR) [24].

\section{Hemodynamic Instability}

Most children experienced shock syndrome that is refractory to volume resuscitation, which ultimately mandates vasopressor support. A case series from the UK by Riphagen et al. found that during 10 days in mid-April 2020, eight children who were previously fit and were tested negative for SARSCoV-2 experienced a hyperinflammatory syndrome with multiorgan involvement similar to Kawasaki disease shock 
syndrome (KDSS), which eventually developed into a vasoplegic shock, and need to be referred to the PICU [10]. A longitudinal national cohort among UK children with PIMS-TS by Davies et al. demonstrated that the incidence of PIMS-TS requiring intensive care would be 1-5\% [12]. Previously, hemodynamic instability has been reported in adults with SARS-CoV-2 sepsis. All of them met the clinical criteria for shock, and were positive on SARS-CoV-2 nasal swab PCR and predominantly pulmonary, renal, hepatic, and cardiac involvement, as well as coagulopathy disorder. Meanwhile, children presenting with PIMS-TS had no remarkable pulmonary and renal symptoms, with none but one coagulopathy parameter elevated, i.e., D-dimer levels. Therefore, mark differentiation exists between adults with viral sepsis and children with PIMS-TS [12].

\section{Mucocutaneous Features}

Both PIMS-TS and KD share similarities regarding mucocutaneous inflammation $[6,7]$. Maculopapular rash, conjunctivitis, and cheilitis are the most common clinical mucocutaneous features found in children with PIMS-TS. Nonetheless, there were no further detailed descriptions of the mucocutaneous lesion, including the onset or location predisposition of PIMS-TS compared to KD. Of note, the presence of skin lesions and other symptoms, in particular abdominal pain, increases the susceptibility of PIMS-TS [8]. Table 3 describes mucocutaneous features from PIMS-TS children $[2,3,8$, $10-12,14,17,18,20]$.

\section{Gastrointestinal Symptoms}

PIMS-TS patients predominantly had gastrointestinal symptoms, in contrast to KD. More than $50 \%$ of patients with PIMS-TS experienced abdominal pain, nausea, vomiting, or diarrhea, in addition to fever. The detailed characterization of the abdominal pain has not been described yet, although the degree of pain is probably severe. A report from Toubiana et al. found that among 21 patients with Kawasaki-like multisystem inflammatory syndrome, all patients had gastrointestinal symptoms, which occurred early in the course of illness, consisting of acute abdominal pain, and $95 \%$ is associated with vomiting and diarrhea. In addition, four patients had peritoneal effusion, which two of them underwent surgery because of an acute abdomen. Moreover, among patients who had diarrhea, all experienced non-bloody diarrhea [10].

A case series from a tertiary hospital in Spain by CabreroHernandez et al. described five patients that came to the Emergency Department (ED) with a history of 2-5 days of abdominal pain and vomiting. Three of them localized the pain in the right iliac fossa. Eventually, they experienced hemodynamic instability and thus were admitted to the PICU due to suspected abdominal sepsis. All patients underwent abdominal ultrasound/computed tomography (CT) and revealed intestinal inflammation without any surgically treatable disease. No microbiologic confirmation of bacterial infection was obtained from the collected cultures. On admission, the SARS-CoV-2 PCR testing with a nasal swab was negative in 4 out of 5 patients. Meanwhile, despite negative PCR results, all patients were treated with SARS-CoV-2 empirical treatment and were discharged after 2-13 days of PICU admission. Before the discharge, patients were positive for IgG SARSCoV-2 $(n / N=2 / 5)$ and SARS-CoV-2 PCR $(n / N=2 / 5)$ [8]. Thus, these findings substantiate post-immunological reaction as the trigger of the hyperinflammatory syndrome with gastrointestinal symptoms as one of the most common chief complaints. The proportion of gastrointestinal symptoms in PIMSTS children is described in Table $4[2,3,8,10,11,13,14$, 16-19].

\section{Cardiac Involvement}

Cardiac involvement in COVID-19 and PIMS-TS might be explained by the accumulation of genetic susceptibility, myocardial injury induced by cytokine storm, and direct viral invasion of SARS-CoV-2 into cardiac myocytes [11, 14]. PIMS-TS is commonly found in children with African descent, which might be of higher risk of COVID-19-related cardiac complications because of intrinsic genetic susceptibility, p.Ser1103TyrSCN5A, which predisposes them to ventricular arrhythmia and sudden cardiac death [25]. The post-viral immunological reaction is postulated to be the culprit of myocardial dysfunction. Evidently, following the administration of IVIG, there was a significant recovery of myocardial dysfunction and a decrease in inflammatory biomarkers.

In a tertiary hospital case series of 20 critically ill children with PIMS-TS in Paris, France, patients had acute myocarditis that was less severe than the usual myocarditis. Furthermore, they exhibit similar features of KD and vasoplegia. They also had intense systemic inflammation, in contrast with acute myocarditis due to KD shock syndrome manifested with higher platelet and neutrophil count, and CRP levels. Nonetheless, the underlying mechanism remains obscured as none of them underwent biopsy [18]. Elevated troponin and B-type natriuretic peptide levels are common in patients with cardiac involvement, and most have elevations in C-reactive protein, ferritin, lactate dehydrogenase, and D-dimers. Patients with PIMS-TS tends to be older, with more significant inflammation and myocardial injury than patients with KD. Regarding the differences, laboratory features between PIMS-TS and KD, on top of the discrepancy in the clinical profiles, support evidence that PIMS-TS is a new clinical entity [3]. 
Table 3 Mucocutaneous findings in PIMS-TS

\begin{tabular}{|c|c|c|}
\hline Study & Findings & $n / N(\%)$ \\
\hline \multirow[t]{3}{*}{ Toubiana et al. [2] } & Rash & $16 / 21(76)$ \\
\hline & Bilateral bulbar conjunctival injection & $17 / 21(81)$ \\
\hline & Lips and oral cavity changes & $16 / 21(76)$ \\
\hline \multirow[t]{4}{*}{ Whittaker et al. [3] } & Erythematous rash & $30 / 58(52)$ \\
\hline & Conjunctivitis & $26 / 58(45)$ \\
\hline & Mucus membrane changes and red cracked lips & $17 / 58(29)$ \\
\hline & Swollen hands and feet & $9 / 58(16)$ \\
\hline \multirow[t]{2}{*}{ Cabrero-Hernandez et al. [8] } & Maculopapular rash in genital & $1 / 5(20)$ \\
\hline & Maculopapular rash in trunk and extremities & $1 / 5(20)$ \\
\hline \multirow[t]{2}{*}{ Riphagen et al. [10] } & Conjunctivitis & $5 / 8(63)$ \\
\hline & Rash & $4 / 8(50)$ \\
\hline \multirow[t]{3}{*}{ Kaushik et al. [11] } & Mucocutaneous involvement & $7 / 33(21)$ \\
\hline & Conjunctivitis & $12 / 33(36)$ \\
\hline & Rash & $14 / 33(42)$ \\
\hline \multirow[t]{2}{*}{ Davies et al. [12] } & Conjunctivitis & $23 / 78(29)$ \\
\hline & Rash & $35 / 78(45)$ \\
\hline \multirow[t]{4}{*}{$\mathrm{Ng}$ et al. [14] } & Conjunctivitis & $3 / 3(100)$ \\
\hline & Maculopapular rash & $1 / 3(33)$ \\
\hline & Urticarial rash & $1 / 3(33)$ \\
\hline & Cracked lips & $1 / 3(33)$ \\
\hline \multirow[t]{4}{*}{ Pouletty et al. [17] } & Skin rash & $13 / 16(81)$ \\
\hline & Hands/feet erythema & $11 / 16(68)$ \\
\hline & Conjunctivitis & $15 / 16(94)$ \\
\hline & Dry cracked lips & $14 / 16(87)$ \\
\hline \multirow[t]{3}{*}{ Grimaud et al. [18] } & Skin rash & $10 / 20(50)$ \\
\hline & Conjunctivitis & $6 / 20(30)$ \\
\hline & Cheilitis & $5 / 20(25)$ \\
\hline \multirow[t]{3}{*}{ Cheung et al. [20] } & Rash & $12 / 17(71)$ \\
\hline & Conjunctivitis & $11 / 17(65)$ \\
\hline & Lip redness/swelling & 9/17 (53) \\
\hline
\end{tabular}

In addition to myocardial dysfunction, children with PIMSTS were also reported to have risks of developing coronary artery aneurysm $[3,7,13,15-18]$. However, a study from
Whittaker et al. among 58 children with PIMS-TS in the UK showed no association between the inflammation markers' levels and the development of coronary artery aneurysm. This
Table 4 Gastrointestinal symptoms in PIMS-TS

\begin{tabular}{llll}
\hline Study & Abdominal Pain, $n / N(\%)$ & Diarrhea, $n / N(\%)$ & Vomiting, $n / N(\%)$ \\
\hline Toubiana et al. [2] & $21 / 21(100)$ & $20 / 21(95)$ & $20 / 21(95)$ \\
Whittaker et al. [3] & $31 / 58(53)$ & $30 / 58(52)$ & $26 / 58(45)$ \\
Cabrero-Hernandez et al. [8] & $5 / 5(100)$ & $3 / 5(60)$ & $5 / 5(100)$ \\
Riphagen et al. [10] & $6 / 8(75)$ & $7 / 8(87.5)$ & $3 / 8(37.5)$ \\
Kaushik et al. [11] & $21 / 33(63)$ & $16 / 33(48)$ & $23 / 33(69)$ \\
Ramcharan et al. [13] & $13 / 15(87)^{*}$ & & $3 / 3(100)$ \\
Ng et al. [14] & $3 / 3(100)$ & $3 / 3(100)$ & $2 / 4(50)$ \\
Blondiaux et al. [16] & $4 / 4(100)$ & $2 / 4(50)$ & $20 / 20(100)$ \\
Pouletty et al. [17] & $13 / 16(81)^{*}$ & & \\
Grimaud et al. [18] & $20 / 20(100)$ & N/A & \\
Capone et al. [19] & $32 / 33(97)^{*}$ & & \\
\hline
\end{tabular}

*These studies did not provide any detailed descriptions regarding the gastrointestinal symptoms 
Table 5 Cardiac involvement in PIMS-TS [2, 3, 7, 9-11, 13, 15-19, 23, 27]

\begin{tabular}{llr}
\hline Cardiac manifestations & $n / N$ & $\%$ \\
\hline Pericardial effusion & $53 / 261$ & 20.3 \\
Ventricular arrhythmia & $4 / 62$ & 6.5 \\
Mitral regurgitation & $22 / 261$ & 8.4 \\
Tricuspid regurgitation & $9 / 261$ & 3.5 \\
Myocarditis & $23 / 261$ & 8.8 \\
Pericarditis & $4 / 261$ & 1.5 \\
LVEF < 55\% & $179 / 258$ & 69.3 \\
Coronary arteries dilatation $(Z$-score of $2-2.5)$ & $23 / 196$ & 11.7 \\
Coronary arteries aneurysm $(Z$-score of $>2.5)$ & $18 / 196$ & 9.2 \\
Cardiac arrest [11] & $1 / 274$ & 0.004 \\
\hline
\end{tabular}

finding suggests that the severity of inflammation does not solely predicate the coronary artery changes. Moreover, this underscores the importance of routine echocardiographic tests to detect any coronary aneurysm across the spectrum of PIMSTS [3]. Among children with abnormal coronary artery without objective dilatation, there would be an "echo-bright" visualization on the echocardiography, which might be explained by the presence of coronary arteries' endothelial inflammation [2, 10, 13]. Therefore, careful follow-up of PIMS-TS patients with this finding is warranted.

Another single-center study by Ramcharan et al. among 15 children with PIMS-TS in a UK tertiary pediatric hospital found 13 patients $(87 \%)$ that had atrioventricular valve regurgitation (10 patients with mitral regurgitation) during admission with an improvement in 1-2 days, which suggested a transient valvulitis [13].

To date, there has been only one case series reporting the description of PIMS-TS' cardiac MRI. The result showed diffuse myocardial edema on T2-STIR sequences and native-T1 mapping, without the evidence of fibrosis replacement or focal necrosis [16]. This finding also supported the hypothesis of the post-viral immune response as the potential mechanism for PIMS-TS, which is similar to KD, due to the presence of macrophages and neutrophil infiltration in the myocardial interstitial, in contrast with myocardial cell degeneration as occurring in viral myocarditis $[16,26]$. We compiled the cardiac involvement manifestations of PIMS-TS from case series and case reports in Table 5.

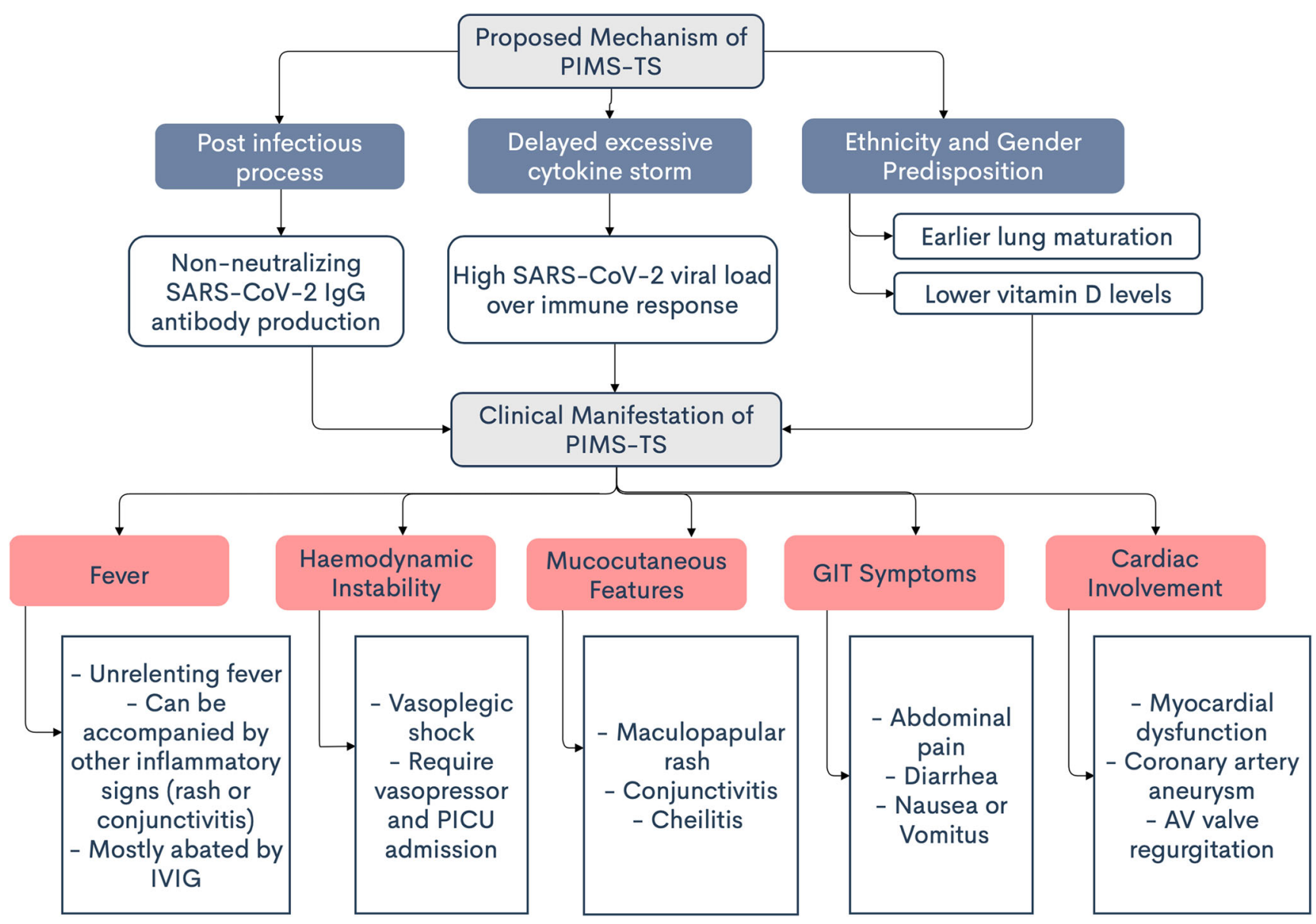

Fig. 1 Clinical manifestations and characteristic of PIMS-TS 


\section{Clinician's Role in Differentiating PIMS-TS}

The role of clinicians during this pandemic is highly crucial in order to recognize its signs and symptoms. According to the age group, PIMS-TS patients tend to be older than KD, which is 8 up to 15 years old; meanwhile, KD typically affects infant or children under 5 years old. Inflammation signs and symptoms of PIMS-TS are mostly similar with KD's symptoms. However, gastrointestinal symptoms such as abdominal pain, nausea, vomiting, and diarrhea are more predominant in PIMS-TS. Myocardial dysfunction and shock more commonly occur in PIMS-TS in comparison with classic KD. Both diseases have almost the same laboratory abnormalities; however, D-dimer and ferritin levels in PIMS-TS are mostly found higher than those in KD.

Considering the high prevalence of asymptomatic SARSCoV-2 infection patients amid this pandemic, thus, if clinicians are faced toward children with any symptoms similar to $\mathrm{KD}$, especially with predominance of gastrointestinal symptoms, hence, PIMS-TS should be emerged as one of the differential diagnoses.

\section{Conclusion}

PIMS-TS is an essential clinical syndrome that resembles atypical/typical KD and presents with a broad clinical spectrum, including cardiac complications. In contrast with $\mathrm{KD}$, PIMS-TS appears to occur in children at an older age with a predominance of gastrointestinal symptoms, hemodynamic instability, and myocardial dysfunction. However, the exact pathomechanism remains to be understood. The management of PIMS-TS is quite complicated due to the broad spectrum of patients. The treatment to prevent the progression of the disease still needs to be validated.

Further investigation and cardiac follow-up should be considered in children with self-resolving inflammation with risk for coronary artery aneurysms. Nevertheless, the post-viral immunological reaction is postulated to be the underlying mechanistic underpinnings. The multifaceted nature of the course of PIMS-TS underlines the need for early recognition and multispecialty care and management. Fortunately, the survival rate is relatively high. Nonetheless, further studies are needed to depict the long-term prognosis, especially relating to coronary artery aneurysm in PIMS-TS. The proposed mechanism and clinical manifestations of PIMS-TS are summarized in Fig. 1.

Authors' Contribution SL conceived and designed the article. SL and JH performed the literature research, acquired the data, and drafted the manuscript. EW, LPS, AS, and CJC contributed in the revision of the manuscript, supervision, and final approval of the manuscript. All authors approved the final manuscript.

\section{Compliance with Ethical Standards}

Conflict of Interest The authors declare that they have no competing interests.

\section{References}

1. World Health Organization. Coronavirus disease (COVID-19) situation reports 190 [Internet] Available at: https://www.who.int/ emergencies/diseases/novel-coronavirus-2019/situation-reports. Accessed 14 Sept 2020.

2. Toubiana J, Poirault C, Corsia A, Bajolle F, Fourgeaud J, Angoulvant F, et al. Kawasaki-like multisystem inflammatory syndrome in children during the covid-19 pandemic in Paris, France: prospective observational study. BMJ [Internet]. 2020;369. Available from: https://www.bmj.com/content/369/bmj.m2094. Accessed 20 Jul 2020.

3. Whittaker E, Bamford A, Kenny J, Kaforou M, Jones CE, Shah P, et al. Clinical characteristics of 58 children with a pediatric inflammatory multisystem syndrome temporally associated with SARSCoV-2. JAMA. 2020;324(3):259-69.

4. World Health Organization. Multisystem inflammatory syndrome in children and adolescents temporally related to COVID-19 [Internet]. [cited $2020 \mathrm{Jul}$ 29]. Available from: https://www.who. int/news-room/commentaries/detail/multisystem-inflammatorysyndrome-in-children-and-adolescents-with-covid-19.

5. Centers for Disease Control and Prevention. Multisystem inflammatory syndrome in children (MIS-C) associated with coronavirus disease 2019 (COVID-19) [Internet]. 2020. Available from: https:// emergency.cdc.gov/han/2020/han00432.asp. Accessed 20 Jul 2020.

6. Royal College of Paediatrics and Child Health Guidance: Paediatric multisystem inflammatory syndrome temporally associated with COVID-19 [Internet]. RCPCH. Available from: https://www. rcpch.ac.uk/resources/guidance-paediatric-multisysteminflammatory-syndrome-temporally-associated-covid-19-pims. Accessed 20 Jul 2020.

7. Verdoni L, Mazza A, Gervasoni A, Martelli L, Ruggeri M, Ciuffreda M, et al. An outbreak of severe Kawasaki-like disease at the Italian epicentre of the SARS-CoV-2 epidemic: an observational cohort study. Lancet. 2020;395(10239):1771-8.

8. Cabrero-Hernández M, García-Salido A, Leoz-Gordillo I, AlonsoCadenas JA, Gochi-Valdovinos A, González Brabin A, et al. Severe SARS-CoV-2 infection in children with suspected acute abdomen: a case series from a tertiary hospital in Spain. Pediatr Infect Dis J. 2020;39(8):e195-8.

9. Rauf A, Vijayan A, John ST, Krishnan R, Latheef A. Multisystem inflammatory syndrome with features of atypical Kawasaki disease during COVID-19 pandemic. Indian J Pediatr. 2020. https://doi. org/10.1007/s12098-020-03357-1.

10. Riphagen S, Gomez X, Gonzalez-Martinez C, Wilkinson N, Theocharis P. Hyperinflammatory shock in children during COVID-19 pandemic. Lancet Lond Engl. 2020;395(10237): 1607-8.

11. Kaushik S, Aydin SI, Derespina KR, Bansal PB, Kowalsky S, Trachtman R, et al. Multisystem inflammatory syndrome in children associated with severe acute respiratory syndrome coronavirus 2 infection: a multi-institutional study from New York City. J Pediatr. 2020;224:24-9.

12. Davies P, Evans C, Kanthimathinathan HK, Lillie J, Brierley J, Waters $\mathrm{G}$, et al. Intensive care admissions of children with paediatric inflammatory multisystem syndrome temporally associated with SARS-CoV-2 (PIMS-TS) in the UK: a multicentre observational 
study. Lancet Child Adolesc Health. 2020. https://doi.org/10.1016/ S2352-4642(20)30215-7.

13. Ramcharan T, Nolan O, Lai CY, Prabhu N, Krishnamurthy R, Richter AG, et al. Paediatric inflammatory multisystem syndrome: temporally associated with SARS-CoV-2 (PIMS-TS): cardiac features, management and short-term outcomes at a UK tertiary paediatric hospital. Pediatr Cardiol. 2020:1-11.

14. Ng KF, Kothari T, Bandi S, Bird PW, Goyal K, Zoha M, et al. COVID-19 multisystem inflammatory syndrome in three teenagers with confirmed SARS-CoV-2 infection. J Med Virol [Internet]. [cited $2020 \mathrm{Jul}$ 29]. Available from: https://onlinelibrary.wiley. com/doi/abs/10.1002/jmv.26206

15. Belhadjer Z, Méot M, Bajolle F, Khraiche D, Legendre A, Abakka $\mathrm{S}$, et al. Acute heart failure in multisystem inflammatory syndrome in children (MIS-C) in the context of global SARS-CoV-2 pandemic. Circulation [Internet]. [cited $2020 \mathrm{Jul}$ 29]. Available from: https://www.ahajournals.org/doi/10.1161/CIRCULATIONAHA. 120.048360 .

16. Blondiaux E, Parisot P, Redheuil A, Tzaroukian L, Levy Y, Sileo $\mathrm{C}$, et al. Cardiac MRI of children with multisystem inflammatory syndrome (MIS-C) associated with COVID-19: case series. Radiology. 2020. https://doi.org/10.1148/radiol.2020202288.

17. Pouletty M, Borocco C, Ouldali N, Caseris M, Basmaci R, Lachaume $\mathrm{N}$, et al. Paediatric multisystem inflammatory syndrome temporally associated with SARS-CoV-2 mimicking Kawasaki disease (Kawa-COVID-19): a multicentre cohort. Ann Rheum Dis. 2020;79(8):999-1006.

18. Grimaud M, Starck J, Levy M, Marais C, Chareyre J, Khraiche D, et al. Acute myocarditis and multisystem inflammatory emerging disease following SARS-CoV-2 infection in critically ill children. Ann Intensive Care. 2020;10(1):69.

19. Capone CA, Subramony A, Sweberg T, Schneider J, Shah S, Rubin $\mathrm{L}$, et al. Characteristics, cardiac involvement, and outcomes of multisystem inflammatory disease of childhood (MIS-C) associated with SARS-CoV-2 infection. J Pediatr [Internet]. 2020; Available from: https://www.ncbi.nlm.nih.gov/pmc/articles/PMC7293762/. Accessed 20 Jul 2020.
20. Cheung EW, Zachariah P, Gorelik M, Boneparth A, Kernie SG, Orange JS, et al. Multisystem inflammatory syndrome related to COVID-19 in previously healthy children and adolescents in New York City. JAMA. 2020;324(3):294-6.

21. Rowley AH. Understanding SARS-CoV-2-related multisystem inflammatory syndrome in children. Nat Rev Immunol. 2020;20(8): 453-4.

22. Rapid risk assessment: Paediatric inflammatory multisystem syndrome and SARS-CoV-2 infection in children [Internet]. European Centre for Disease Prevention and Control. 2020 [cited 2020 Jul 29]. Available from: https://www.ecdc.europa.eu/en/ publications-data/paediatric-inflammatory-multisystem-syndromeand-sars-cov-2-rapid-risk-assessment.

23. Dasgupta K, Finch SE. A case of pediatric multisystem inflammatory syndrome temporally associated with COVID-19 in South Dakota. S D Med. 2020;73(6):246-51.

24. Canadian Paediatric Society. Paediatric inflammatory multisystem syndrome temporally associated with COVID-19 | Canadian Paediatric Society [Internet]. Available from: https://www.cps.ca/ en/documents/position/pims. Accessed 5 Aug 2020.

25. Giudicessi JR, Roden DM, Wilde AAM, Ackerman MJ. Genetic susceptibility for COVID-19-associated sudden cardiac death in African Americans. Heart Rhythm [Internet]. 2020; Available from: https://www.ncbi.nlm.nih.gov/pmc/articles/PMC7198426/. Accessed 29 Jul 2020.

26. Harada M, Yokouchi Y, Oharaseki T, Matsui K, Tobayama H, Tanaka N, et al. Histopathological characteristics of myocarditis in acute-phase Kawasaki disease. Histopathology. 2012;61(6): 1156-67.

27. Greene AG, Saleh M, Roseman E, Sinert R. Toxic shock-like syndrome and COVID-19: a case report of multisystem inflammatory syndrome in children (MIS-C). Am J Emerg Med [Internet]. 2020; Available from: https://www.ncbi.nlm.nih.gov/pmc/articles/ PMC7274960/. Accessed 29 Jul 2020.

Publisher's Note Springer Nature remains neutral with regard to jurisdictional claims in published maps and institutional affiliations. 